\title{
Continued Fractions and Linear Recurrences
}

\author{
By W. H. Mills
}

\begin{abstract}
Let $t_{0}, t_{1}, t_{2}, \cdots$ be a sequence of elements of a field $F$. We give a continued fraction algorithm for $t_{0} x+t_{1} x^{2}+t_{2} x^{3}+\cdots$. If our sequence satisfies a linear recurrence, then the continued fraction algorithm is finite and produces this recurrence.
\end{abstract}

More generally the algorithm produces a nontrivial solution of the system

$$
\sum_{j=0}^{s} t_{i+j} \lambda_{j}, \quad 0 \leqslant i \leqslant s-1,
$$

for every positive integer $s$.

1. Let $t_{0}, t_{1}, t_{2}, \cdots$ be a sequence of elements of a field $F$. Set

$$
T=\sum_{j=0}^{\infty} t_{\dot{j}^{j}} x^{j}
$$

Let $d$ be a nonnegative integer. We say that $T^{*}$ is an approximation of $T$ of degree $d$ if there exist polynomials $V$ and $W$ such that $T^{*}=V / W, \operatorname{deg} V<d$, $\operatorname{deg} W \leqslant d, x \nmid W$, and $x^{2 d} \mid W T-V$.

We shall give a continued fraction expansion for $x T$. This yields polynomials $V_{i}, W_{i}$, and integers $d_{i}, 0=d_{1}<d_{2}<d_{3}<\cdots$, such that $\left(V_{i}, W_{i}\right)=1$ and $V_{i} / W_{i}$ is an approximation of $T$ of degree $d_{i}$. Suppose $T^{*}$ is any approximation of $T$ of some degree $d$. Then $T^{*}=V_{i} / W_{i}$ for that value of $i$ such that $d_{i} \leqslant$ $d<d_{i+1}$.

If the sequence of the $t_{j}$ satisfies a linear recurrence of degree $d$, but not one of smaller degree, then there is an $m$ such that $d_{m}=d$ and the linear recurrence is given by the polynomial $W_{m}$. In this case, $W_{m} T=V_{m}$, the continued fraction expansion, terminates at $i=m$, and we can determine $W_{m}$ from the first $2 d$ of the $t_{j}$, i.e., from those $t_{j}$ such that $0 \leqslant j<2 d$.

Our algorithm is closely related to Zierler's version of Berlekamp's algorithm [1].

2. We consider continued fraction expansions of the form

$$
\alpha=N_{1}+\frac{1}{N_{2}+\frac{1}{N_{3}+\cdots}},
$$

where $N_{1}, N_{2}, N_{3}, \cdots$ are elements from some field $E$. We can write

Received January 28, 1974.

AMS (MOS) subject classifications (1970). Primary 12C10, $10 \mathrm{~F} 20$. 


$$
\alpha=N_{1}+R_{1}, \quad 1 / R_{1}=N_{2}+R_{2}, \quad 1 / R_{2}=N_{3}+R_{3}, \cdots .
$$

If $R_{m}=0$ for some $m$, then the continued fraction terminates with $N_{m}$. Otherwise it is an infinite continued fraction.

In the classical case, $\alpha$ is a real number, the $N_{i}$ are integers, and $0 \leqslant R_{i}<1$ for all $i$. We are interested in a different case.

We set

$$
\begin{gathered}
P_{0}=1, \quad Q_{0}=0 ; \quad P_{1}=N_{1}, \quad Q_{1}=1, \\
P_{i}=N_{i} P_{i-1}+P_{i-2}, \quad i \geqslant 2,
\end{gathered}
$$

and

$$
Q_{i}=N_{i} Q_{i-1}+Q_{i-2}, \quad i \geqslant 2
$$

It is well known, and easy to show, that

$$
\begin{aligned}
& P_{1} / Q_{1}=N_{1}, \quad P_{2} / Q_{2}=N_{1}+1 / N_{2}, \\
& P_{3} / Q_{3}=N_{1}+1 /\left(N_{2}+1 / N_{3}\right), \cdots .
\end{aligned}
$$

The sequence $P_{1} / Q_{1}, P_{2} / Q_{2}, P_{3} / Q_{3}, \cdots$ converges to $\alpha$ in many cases, including the classical case.

We put

$$
\Delta_{i}=\alpha Q_{i}-P_{i}, \quad i \geqslant 0
$$

Then we have

$$
\Delta_{0}=-1, \quad \Delta_{1}=\alpha-N_{1}
$$

and

$$
\Delta_{i}=N_{i} \Delta_{i-1}+\Delta_{i-2}, \quad i \geqslant 2 .
$$

Clearly $R_{1}=\alpha-N_{1}=-\Delta_{1} / \Delta_{0}$. Since $R_{i+1}=-N_{i+1}+1 / R_{i}$ it follows from (5), by induction on $i$, that

$$
R_{i}=-\Delta_{i} / \Delta_{i-1}, \quad i \geqslant 1
$$

3. We now take $E$ to be the field of all series of the form $\sum_{j=k}^{\infty} a_{j} x^{j}$, where the $a_{j}$ are elements of the field $F$ and $k$ is a rational integer which may be negative. For convenience let $y=1 / x$. We set $\alpha=x T$ and $N_{1}=0$. Then $R_{1}=$ $\alpha=x T$. We now define the $N_{i}$ and $R_{i}$ inductively using

$$
1 / R_{i-1}=N_{i}+R_{i}, \quad i \geqslant 2,
$$

where we take $N_{i}$ to be a polynomial in $y$ and $x \mid R_{i}$. Thus if

$$
1 / R_{i-1}=\sum_{j=k}^{\infty} a_{j} x^{j}, \quad a_{k} \neq 0,
$$


it turns out that $k<0$ and we have

$$
N_{i}=\sum_{j=k}^{0} a_{j} x^{j}=\sum_{u=0}^{-k} a_{-u} y^{u} \quad \text { and } \quad R_{i}=\sum_{j=1}^{\infty} a_{j} x^{j} .
$$

This determines the $N_{i}$ and $R_{i}$ uniquely. If $R_{m}=0$ for some $m$, then the process terminates at this point. The $P_{i}, Q_{i}$, and $\Delta_{i}$ are now determined by (1), (2), (3), (4), and (5).

We shall write $x^{r} \| A$ if $x^{r}$ divides $A$, but $x^{r+1}$ does not divide $A$. This means that $A$ is of the form $A=\sum_{j=r}^{\infty} a_{j} x^{j}$ with $a_{r} \neq 0$. Let $x^{r_{i}} \| R_{i}, i \geqslant 1$. If $R_{m}=0$, we set $r_{m}=\infty$. Then $r_{i} \geqslant 1$ for $i \geqslant 1$. For $i \geqslant 2, N_{i}$ is a polynomial in $y$ of degree $r_{i-1}$. Set

$$
d_{i}=\sum_{j=1}^{i-1} r_{j}
$$

Then we have $0=d_{1}<d_{2}<d_{3}<\cdots$. It follows from (1) and (3), by induction on $i$, that $Q_{i}$ is a polynomial in $y$ of degree $d_{i}$. Similarly, for $i \geqslant 2, P_{i}$ is a polynomial in $y$ of degree $d_{i}-r_{1}$. Set

$$
V_{i}=x^{d_{i}^{-1}} P_{i}, \quad W_{i}=x^{d_{i}} Q_{i} .
$$

Then $V_{i}$ and $W_{i}$ are polynomials in $x, \operatorname{deg} V_{i}<d_{i}$, and $\operatorname{deg} W_{i} \leqslant d_{i}$. Moreover, $w_{i}$ has a nonzero constant term so that $x \nmid w_{i}$. Now

$$
T W_{i}-V_{i}=x^{d_{i}-1}\left(\alpha Q_{i}-P_{i}\right)=x^{d_{i}^{-1}} \Delta_{i} .
$$

Since $\Delta_{0}=-1,(6)$ gives us

$$
\Delta_{i}=(-1)^{i+1} \prod_{j=1}^{i} R_{j}
$$

Since $x^{r_{j}} \| R_{j}$, we have

$$
x^{d i+1} \| \Delta_{i}
$$

by (8). Hence

$$
x^{d_{i}+d_{i+1}^{-1}} \| T W_{i}-V_{i}
$$

Therefore, $x^{2 d_{i}} \mid T W_{i}-V_{i}$ so that $V_{i} / W_{i}$ is an approximation of $T$ of degree $d_{i}$.

Lemma 1. Let $T^{*}$ be an approximation of $T$ of degree $d$. Let $i$ be the integer such that $d_{i} \leqslant d<d_{i+1}$. Then $T^{*}=V_{i} / W_{i}$.

Proof. We have $T^{*}=V / W$, where $\operatorname{deg} W \leqslant d, \operatorname{deg} V<d$, and $x^{2 d} \mid W T-V$. Now $d+d_{i} \leqslant 2 d$ so that $x^{d+d_{i}} \mid W T-V$. Moreover, $d+d_{i} \leqslant d_{i}+d_{i+1}-1$ so that $x^{d+d_{i}} \mid W_{i} T-V_{i}$ by (10). Since

$$
V_{i} W-V W_{i}=W_{i}(W T-V)-W\left(W_{i} T-V_{i}\right),
$$


we have

$$
x^{d+d_{i}} V_{i} W-V w_{i}
$$

Now the degree of $V_{i} W-V W_{i}$ is less than $d+d_{i}$. Therefore $V_{i} W-V W_{i}=0$, so that

$$
T^{*}=V / W=V_{i} / W_{i}
$$

Lemma 2. If $V_{i} / w_{i}=V_{j} / w_{j}$, then $i=j$.

Proof. Suppose $V_{i} / W_{i}=V_{j} / W_{j}$. Then we have $V_{i}=V D, w_{i}=W D, V_{j}=V E$, $W_{j}=W E$ for suitable polynomials $V, W, D, E$ with $(V, W)=1$. Since $x \nmid w_{i}$, we have $x \nmid D$ so that (10) yields

Similarly

$$
x^{d_{i}+d_{i+1}-1} \| T W-V
$$

$$
x^{d_{j}+d_{j+1}^{-1}} \| T W-V
$$

Hence

$$
d_{i}+d_{i+1}-1=d_{j}+d_{j+1}-1
$$

Therefore, $i=j$.

Lemma 3. $\left(V_{i}, W_{i}\right)=1$.

Proof. Suppose $\left(V_{i}, W_{i}\right)=D$ where $\operatorname{deg} D>0$. Then $V_{i}=V D, W_{i}=W D$ for suitable polynomials $V, W$ such that $x \nmid W, \operatorname{deg} W<d_{i}$, and $\operatorname{deg} V<d_{i}-1$. Moreover $x \nmid D$ so that $x^{2 d_{i}} \mid T W-V$. Hence $V / W$ is an approximation of $T$ of degree less than $d_{i}$. By Lemma 1 we have $V / W=V_{j} / W_{j}$ for some $j<i$. This contradicts Lemma 2.

Lemma 4. For any particular value of $i$ we have either $\operatorname{deg} V_{i}=d_{i}-1$ or $\operatorname{deg} W_{i}=d_{i}$.

Proof. Since $\operatorname{deg} W_{1}=0=d_{1}$, we may suppose $i>1$. If the result is false, then $V_{i} / W_{i}$ is an approximation of $T$ of degree less than $d_{i}$. By Lemma 1 this implies that $V_{i} / W_{i}=V_{j} / W_{j}$ for some $j<i$, which contradicts Lemma 2.

4. Let $\left\{t_{j}\right\}=\left\{t_{0}, t_{1}, \cdots, t_{n-1}\right\}$ be a finite sequence of elements of $F$, and set

$$
T=\sum_{j=0}^{n-1} t_{j} x^{j} .
$$

Let $W$ be a polynomial of degree $s$ with a nonzero constant term. Thus $W=$ $\Sigma_{j=0}^{s} w_{j} x^{j}$, where the $w_{j}$ are elements of $F, w_{0} \neq 0, w_{s} \neq 0$. The linear recurrence given by $W$ is

$$
\sum_{i=0}^{s} w_{i} t_{k-i}=0 .
$$

If (11) holds for a particular value $k_{0}$ of $k$, we say that the linear recurrence $W$ holds 
for $k_{0}$. If (11) holds for all values of $k$ for which the left side is defined, i.e., for $s \leqslant k \leqslant n-1$, then we say that the sequence $\left\{t_{j}\right\}$ satisfies the linear recurrence $W$.

Whenever we speak of a linear recurrence $W$ we shall mean a polynomial $W$ with a nonzero constant term. The degree of the linear recurrence is defined to be the degree of this polynomial.

In order to determine $W$, up to a multiplicative constant, we must have (11) satisfied by at least $s$ values of $k$. Hence we must have $2 s \leqslant n$. Our problem is to determine whether or not the sequence $\left\{t_{j}\right\}$ satisfies a linear recurrence of degree $\leqslant n / 2$, and if so to determine the linear recurrence of lowest degree that $\left\{t_{j}\right\}$ satisfies.

Let $h=[n / 2]$. Thus $h$ is an integer and either $n=2 h$ or $n=2 h+1$. Let $x T$ be expanded in a continued fraction as indicated in Section 2 and Section 3. This gives us polynomials $V_{i}$ and $W_{i}$ and integers $d_{i}$. Let $m$ be the integer such that $d_{m} \leqslant h<d_{m+1}$. This is equivalent to

$$
2 d_{m} \leqslant n<2 d_{m+1} .
$$

Now suppose that the sequence $\left\{t_{j}\right\}$ satisfies a linear recurrence $W$ of degree $s$, where $s \leqslant n / 2$. Thus $s \leqslant h$. We suppose $W$ chosen so that $s$ is minimal. Set $V=\Sigma_{j=0}^{s-1} v_{j} x^{j}$, where

$$
v_{j}=\sum_{i=0}^{j} w_{i} t_{j-i}
$$

Then $x^{n} \mid T W-V$ by (11) so that $V / W$ is an approximation of $T$ of degree $h$. More precisely it is an approximation of $T$ of degree $d$ for any $d$ such that $s \leqslant$ $d \leqslant h$. By Lemma 1 and the choice (12) of $m$ we have $V / W=V_{m} / W_{m}$. Since $W$ is of minimal degree, we have $(V, W)=1$. Moreover $\left(V_{m}, W_{m}\right)=1$ by Lemma 3 , so that $W=\lambda W_{m}$ for some nonzero element $\lambda$ of $F$.

More generally, suppose only that the linear recurrence $W$ holds for those $k$ such that $h \leqslant k \leqslant n-1$, that $\operatorname{deg} W \leqslant h$, and that $W$ is a linear recurrence of minimal degree with these properties. As above there is a polynomial $V$ such that $V / W$ is an approximation of $T$ of degree $h,(V, W)=1$, and $W=\lambda W_{m}$ for some nonzero $\lambda$ in $F$.

It is easy to see that there need not be such a linear recurrence. For example, we may take $\left\{t_{j}\right\}=\{0,0, \cdots, 0,1\}$. However, we have shown that if there is one, then it must be $W_{m}$, up to a multiplicative constant.

Now

$$
x^{d_{m}{ }^{+d}{ }_{m+1}^{-1}} \| T W_{m}-V_{m}
$$

by (10). Hence if $n \geqslant d_{m}+d_{m+1}$, then $\left\{t_{j}\right\}$ does not satisfy the linear recurrence $W_{m}$, in fact $W_{m}$ fails to hold for $d_{m}+d_{m+1}-1$. Thus we have the following result:

Theorem 1. If $d_{m}+d_{m+1} \leqslant n<2 d_{m+1}$, then the sequence $\left\{t_{j}\right\}$ does 
not satisfy any linear recurrence of degree $\leqslant n / 2$. In fact, there is no linear recurrence of degree $\leqslant n / 2$ that holds for all $k$ such that $h \leqslant k \leqslant n-1$.

Now suppose that $n<d_{m}+d_{m+1}$. Then the linear recurrence $W_{m}$ holds for all $k$ in the range $d_{m} \leqslant k \leqslant n-1$. We have $\operatorname{deg} W_{m} \leqslant d_{m}$. If $\operatorname{deg} W_{m}=$ $d_{m}$, then $\left\{t_{j}\right\}$ satisfies the linear recurrence $W_{m}$. However, if $\operatorname{deg} W_{m}<d_{m}$, then $\operatorname{deg} V_{m}=d_{m}-1$ by Lemma 4 , and, therefore, the linear recurrence $W_{m}$ fails to hold at $d_{m}-1$. Thus we have the following result:

Theorem 2. Suppose $2 d_{m} \leqslant n<d_{m}+d_{m+1}$. If $\operatorname{deg} W_{m}=d_{m}$, then $w_{m}$ is a linear recurrence of minimal degree satisfied by $\left\{t_{j}\right\}$. If $\operatorname{deg} W_{m}<d_{m}$, then there is no linear recurrence of degree $\leqslant n / 2$ which is satisfied by $\left\{t_{j}\right\}$. However, $W_{m}$ is a linear recurrence of minimal degree that holds for all $k$ such that $h \leqslant$ $k \leqslant n-1$. It holds for all $k$ in the range $d_{m} \leqslant k \leqslant n-1$, and fails to hold for $d_{m}-1$.

5. In this section, we shall describe an efficient method of computing the polynomial $W_{m}$. As before, let $\left\{t_{j}\right\}=\left\{t_{0}, t_{1}, \cdots, t_{n-1}\right\}$ be the finite sequence we are interested in. We start with $N_{1}=0, \Delta_{0}=-1$, and

$$
\Delta_{1}=x T-N_{1}=\sum_{j=0}^{n-1} t_{j} x^{j+1} .
$$

For $i \geqslant 2,(6)$ and (7) give us

$$
N_{i}+R_{i}=1 / R_{i-1}=-\Delta_{i-2} / \Delta_{i-1}
$$

where $x \mid R_{i}$ and $N_{i}$ is a polynomial in $y, y=1 / x$. Thus $N_{i}$ can be obtained from $\Delta_{i-2}$ and $\Delta_{i-1}$ by an ordinary division process. Then $\Delta_{i}$ is given by (5): $\Delta_{i}=N_{i} \Delta_{i-1}+\Delta_{i-2}$. In this way, the $N_{i}$ and the $\Delta_{i}$ can be successively obtained. We must continue this out to $i=m$ where $2 d_{m} \leqslant n<2 d_{m+1}$. Since $x^{d_{i}} \| \Delta_{i-1}$ by (9), we know at once when we have reached $i=m$. If $d_{m}+d_{m+1} \leqslant n$, then there is no solution. If $d_{m}+d_{m+1}>n$, then we calculate $Q_{m}$ from the $N_{i}$ and the relations $Q_{0}=0, Q_{1}=1, Q_{i}=N_{i} Q_{i-1}+Q_{i-2}$.

If $Q_{m}$ has a nonzero constant term, then $\operatorname{deg} W_{m}=d_{m}$ and $W_{m}=x^{d_{m}} Q_{m}$ is the required linear recurrence. If $Q_{m}$ has no constant term, then $\operatorname{deg} W_{m}<d_{m}$ and $\left\{t_{j}\right\}$ does not satisfy a linear recurrence of degree $\leqslant n / 2$. However, in this case, $W_{m}=x^{d_{m}} Q_{m}$ is a linear recurrence that holds for all $k$ such that $d_{m} \leqslant k \leqslant n-1$. We note that $x^{d_{i}}\left\|\Delta_{i-1}, x^{d_{i-1}}\right\| \Delta_{i-2}$, and $d_{i}=r_{i-1}+d_{i-1}$. Hence in performing the division $\Delta_{i-2} / \Delta_{i-1}$ we need only use the first $r_{i-1}+1$ terms of $\Delta_{i-2}$ and the same number of terms of $\Delta_{i-1}$. This is sufficient to determine $N_{i}$ completely.

Finally we note that it is only necessary to calculate $\Delta_{i}$ out to the term in $x^{n-d_{i}}$. This corresponds to the fact that $\Delta=x T$ is known only out to the term in $x^{n}$. To see this, consider the division of $\Delta_{i-2}$ by $\Delta_{i-1}$. We need $r_{i-1}+1$ terms of each. More terms of $\Delta_{i-2}$ are assumed known than of $\Delta_{i-1}$. The number of terms of $\Delta_{i-1}$ that we have is $n-d_{i-1}-d_{i}+1=n-2 d_{i}+r_{i-1}+1$. Since we 
may suppose $i \leqslant m$, this is at least $r_{i-1}+1$ terms. Thus $N_{i}$ may be computed exactly. Clearly if we know $\Delta_{i-2}$ out to the term in $x^{n-d_{i-2}}$ and $\Delta_{i-1}$ out to the term in $x^{n-d_{i-1}}$, then once $N_{i}$ is known as a polynomial in $y$ of degree $r_{i-1}$, we may calculate $\Delta_{i}$ out to the term in $x^{n-d_{i}}$.

Tables 1 and 2 give examples of the calculation for small $n$ and $F=G F(2)$. The unnecessary terms of $\Delta_{i}$, i.e., those beyond $x^{n-d_{i}}$, are given in parenthesis. In the first example $n=12, m=3, d_{3}=3, d_{4}=7, d_{m}+d_{m+1} \leqslant n$, so there is no solution and the $Q_{i}$ are not calculated. In the second example, the sequence satisfies the linear recurrence $x^{4}+x+1$.

$$
\begin{aligned}
& \text { TABLE } 1 \\
& F=G F(2), n=12,\left\{t_{j}\right\}=\{100101110111\} \\
& i \quad N_{i} \Delta_{i} \\
& \begin{array}{lll}
0 & - & 1 \\
1 & 0 & x+x^{4}+x^{6}+x^{7}+x^{8}+x^{10}+x^{11}+x^{12}
\end{array} \\
& 2 y \quad x^{3}+x^{5}+x^{6}+x^{7}+x^{9}+x^{10}+x^{11} \\
& 3 y^{2}+1 \quad x^{7}\left(+x^{12}\right)
\end{aligned}
$$

There is no linear recurrence of degree $\leqslant 6$.

TABLE 2

$$
F=G F(2), n=8, \quad\left\{t_{j}\right\}=\{11101011\}
$$

\begin{tabular}{llll}
$i$ & \multicolumn{1}{c}{$N_{i}$} & \multicolumn{1}{c}{$\Delta_{i}$} & \multicolumn{1}{c}{$Q_{i}$} \\
0 & - & 1 & 0 \\
1 & 0 & $x+x^{2}+x^{3}+x^{5}+x^{7}+x^{8}$ & 1 \\
2 & $y+1$ & $x^{3}+x^{4}+x^{5}+x^{6}\left(+x^{8}\right)$ & $y+1$ \\
3 & $y^{2}$ & $x^{4}+x^{5}\left(+x^{6}+x^{7}+x^{8}\right)$ & $y^{3}+y^{2}+1$ \\
4 & $y$ & $\left(x^{7}+x^{8}\right)$ & $y^{4}+y^{3}+1$
\end{tabular}

The linear recurrence is $x^{4}\left(y^{4}+y^{3}+1\right)=x^{4}+x+1$.

6. We now consider the system

$$
\sum_{j=0}^{s} t_{i+j} \lambda_{j}, \quad 0 \leqslant i \leqslant s-1,
$$

of $s$ linear equations in $s+1$ unknowns. This system must have at least one nontrivial solution in $F$. If we set

$$
\Lambda=\sum_{j=0}^{s} \lambda_{j} x^{s-j},
$$

then we can write $\Lambda=x^{r} W$, where $W$ is a polynomial with nonzero constant term, 
and $\operatorname{deg} W \leqslant s-r$. If (13) holds, then there is a polynomial $V$ such that $\operatorname{deg} V<$ $s-r$ and $X^{2 s-r} \mid T W-V$. Thus $V / W$ is an approximation of $T$ of degree $s-r$. Hence $V / W=V_{m} / W_{m}$ for some $m$ with $d_{m} \leqslant s-r$ and $d_{m}+d_{m+1}-1 \geqslant$ $2 s-r$, so that $d_{m} \leqslant s<d_{m+1}$. Thus we see that our algorithm can be used to solve the system (13) for any positive integer $s$.

Institute for Defense Analyses

Communications Research Division

Princeton, New Jersey 08540

1. NEAL ZIERLER, "Linear recurring sequences and error-correcting codes," Error Cor recting Codes, edited by H. B. Mann, Wiley, New York, 1968, pp. 47-59. MR 40 \#2438. 